\title{
Kangfuxin promotes apoptosis of gastric cancer cells through activating ER-stress and autophagy
}

\author{
PIAN-PIAN CHEN $^{1 *}$, XIU-YING MA $^{2 *}$, QIAN LIN ${ }^{1}$, HE-LIN XU ${ }^{1}$, HONG-XUE SHI ${ }^{1}$, \\ HONG-YU ZHANG ${ }^{1}$, JIAN XIAO ${ }^{1}$, FU-NENG GENG ${ }^{2}$ and YING-ZHENG ZHAO ${ }^{1}$ \\ ${ }^{1}$ School of Pharmaceutical Sciences, Wenzhou Medical University, Wenzhou, Zhejiang 325035; \\ ${ }^{2}$ Sichuan Key Laboratory of Medical American Cockroach, Chengdu, Sichuan 610000, P.R. China
}

Received March 1, 2017; Accepted September 21, 2017

DOI: $10.3892 / \mathrm{mmr} .2017 .7716$

\begin{abstract}
Gastric cancer is a leading cause of cancer-associated mortality worldwide. In studies on the mechanisms of antigastric cancer drugs, autophagy and endoplasmic reticulum (ER) stress have been demonstrated to serve an active role in gastric cancer. The organic extract of Periplaneta americana (also termed American Cockroach), which is named Kangfuxin (KFX) in China, has been used clinically as a traditional Chinese medicine against disorders, including stomach bleeding, gastric ulcers, tuberculosis, burns and trauma. However, the role of KFX and its mechanism in gastric cancer remains to be elucidated. The present study aimed to determine the effects of $\mathrm{KFX}$ in vitro against cultured the human carcinoma SGC-7901 cell line, and to explore the potential mechanism of the anticancer effects of KFX in gastric cancer. SGC-7901 cells were treated with different concentrations of KFX for varying amounts of time. As a result, KFX treatment decreased the ratio of apoptosis regulators Bcl-2/Bax, activated ER stress and induced significant apoptosis in SGC-7901 cells. Furthermore, KFX was able to restore the ER stress activation blocked by 4-phenylbutyrate. In addition, KFX activated autophagy in SGC-7901 cells. These results demonstrated that ER stress, autophagy and the apoptosis-inducing effects of KFX in SGC-7901 cells may achieve promising anticancer effects in numerous other types of cancer. In particular, ER
\end{abstract}

Correspondence to: Dr Ying-Zheng Zhao, School of Pharmaceutical Sciences, Wenzhou Medical University, 129 Gaojiaoyuan Street, Wenzhou, Zhejiang 325035, P.R. China

E-mail:pharmtds@163.com

Mr. Fu-Neng Geng, Sichuan Key Laboratory of Medical American Cockroach, 88 Yingmenlukou Street, Chengdu, Sichuan 610000, P.R. China

E-mail: haoyishenggfn@126.com

*Contributed equally

Key words: Kangfuxin, SGC-7901 cells, gastric cancer, ER stress, apoptosis, autophagy stress may serve an essential role in KFX-induced anticancer effects on gastric carcinoma and a secondary role in autophagy.

\section{Introduction}

Currently, gastric cancer is the 2nd most common malignant tumour in the world. Tumour resection surgery is the main clinical treatment for curing gastric cancer. However, most patients have a less than five-year survival rate and a poor quality of life after the surgery $(1,2)$. Therefore, the understanding of the biological mechanism of current agents and the development of new therapeutic agents for the clinical management of gastric cancer are still urgent. Complementary and alternative medicine (CAM) is increasingly accepted in United States both as treatment for illness and as self-care to promote health and well-being (3). The use of CAM has increased substantially in conventional and western medical practices over the past decade, notably in cancer treatment (4). CAM includes traditional Chinese medicine (TCM) and acupuncture. TCM has a variety products, such as Chinese herbal medicines, fungi and insects (5). In addition to common Chinese herbal medicines, insects also show potential for alternative therapy (6-8). Periplaneta americana is a species widely disseminated in China and throughout major cosmopolitan areas of the world. Additionally, Periplaneta americana has been exploited as an alternative naturopathic remedy for ulcers, burns, and heart disease in southwestern China and northeastern Brazil (4). As an extract of Periplaneta americana, Kangfuxin (KFX) is a drug approved by the China Food and Drug Administration (CFDA) (Z51021834). The therapeutic effects of KFX include gastric ulcers, tuberculosis, burns and trauma. This study wanted to explore the effects and the mechanism of KFX on gastric cancer therapy. An in vitro model of gastric cancer was set up using the cultured human carcinoma cell lines SGC-7901.

As reported, endoplasmic reticulum (ER) stress is a new pathway that leads to apoptosis, which followed the discovery of the death receptor signalling and mitochondrial pathways (9). Studies reported that stress signals were relayed from the ER to the mitochondria. ER stress-induced apoptosis was similar to mitochondrial-mediated apoptosis (10). ER stress is provoked by the accumulation of unfolded or misfolded proteins in the ER lumen (9). However, prolonged or irreversible ER stress 
switches the adaptive nature of the unfolded protein response (UPR) into a cell death program (11). Moderate ER stress promotes cancer cell survival; however, excessive ER stress leads to cancer apoptosis (10). During excessive ER stress, an apoptotic pathway may be activated via CHOP/GRP78 and caspase-12. Apoptosis in ER stress conditions facilitates cell death, which is also associated with the glucose-regulated protein 78 (GRP78), the transcription activation of the C/EBP homologous transcription factor (CHOP) and the activation of ER-associated caspase-12 (12). Therefore, the overload of ER stress or the blocked UPR can explain the anti-cancer effects of anti-cancer drugs. In addition, autophagy can be induced by ER stress $(13,14)$. To alleviate ER stress, the UPR signalling may activate autophagy to clear the accumulated misfolded proteins from the ER lumen (15).

In this study, we investigated the inhibition of KFX on SGC-7901 cells in vitro and demonstrated whether ER stress and autophagy were involved in its anti-cancer effects. Our data showed that KFX attenuated the survival rate and significantly induced apoptosis in all chemicals. Moreover, the signalling pathways and molecular mechanisms involved in ER stress and autophagy in KFX treated SGC-7901 cells were elucidated. This study supports that KFX is a potential therapy for gastric cancer. ER stress, autophagy and the apoptosis-inducing effects of KFX in cancer cells may have promising anticancer effects in many other cancers.

\section{Materials and methods}

Reagents. KFX (Good Doctor Pharmaceutical Group, Sichuan, China) was dissolved in cell culture medium to the required concentration before use. 4-phenylbutyric acid (4-PBA; Aladdin, Shanghai, China) was dissolved in dimethyl sulfoxide (DMSO) to make a $2 \mathrm{mmol} / 1$ stock solution and was diluted in cell culture medium to the required concentration before use. A FITC-Annexin V Apoptosis detection kit (BD Biosciences, San Jose, CA, USA) were prepared for the apoptosis assay. All chemicals in the present study were used for experimental research only.

Cell culture. A human gastric cancer cell line, SGC-7901, was purchased from the Shanghai Institute of Cell Biology (Shanghai, China). Cells were routinely maintained in RPMI-1640 (31800-022; Gibco; Thermo Fisher Scientific, Inc., Waltham, MA, USA) supplemented with $10 \%$ fetal bovine serum (FBS, SV30087.02; HyClone; GE Healthcare Life Sciences, Logan, UT, USA), penicillin (100 U/ml, P3032) and streptomycin (100 U/ml, S9137) (both from Sigma-Aldrich; Merck Millipore, Darmstadt, Germany) at $37^{\circ} \mathrm{C}$ in a humidified $5 \% \mathrm{CO}_{2}$ incubator.

Cytotoxicity. To detect the anticancer activity of different concentrations of KFX, the SGC-7901 cell viability in different treatment groups was assessed by using a CCK- 8 kit. CCK- 8 was used to measure cell viability and evaluate the survival rate of SGC-7901 cells by KFX treatment. Cells were seeded in a 96-well plate at a density of $2 \times 10^{3}$ cells/well and incubated for $12 \mathrm{~h}$ in $0.1 \mathrm{ml}$ RPMI-1640 medium supplemented with $10 \% \mathrm{FBS}$ at $37^{\circ} \mathrm{C}$. Then, the medium was replaced with fresh medium containing $\mathrm{KFX}$ at various concentrations ranging from 0.1 to $5 \mu \mathrm{g} / \mathrm{ml}$. The untreated cells were used as controls. After 24, 48 and $72 \mathrm{~h}$ incubation, $10 \mu \mathrm{l}$ of CCK-8 was added into each well. After $4 \mathrm{~h}$, absorption in each well was observed by a micro plate reader at $450 \mathrm{~nm}$ (Multiskan MK3; Thermo Fisher Scientific, Inc., Waltham, MA, USA).

Cell migration. SGC-7901 cells were seeded into 6-well plates at $3 \times 10^{5}$ cells/well and were incubated for $12 \mathrm{~h}$. A scratch was created with a sterile clear tip $(200 \mu 1)$. Then, the cells were washed twice with PBS to remove the debris and detached cells. Scratch pictures were recorded immediately using an inverted fluorescence microscope (Nikon, Tokyo, Japan). Afterwards, the cells in the wells were cultured with various concentrations of KFX in medium, separately. The only cells treated with medium were the control group. After 24 or $48 \mathrm{~h}$ of treatment, cell migration was observed by using an inverted fluorescence microscope.

Cell apoptosis assay. SGC-7901 cells were seeded in 6-well plates at a density of $3 \times 10^{5}$ cells/well and incubated overnight at $37^{\circ} \mathrm{C}$. Cells were treated with a different concentration of KFX with the untreated cells as the controls. After $48 \mathrm{~h}$ treatment, the cells were collected and analysed. For quantitative assays, the cells were harvested by trypsinisation, then centrifuged at 1,000 rpm for $3 \mathrm{~min}$ and suspended in binding buffer. After adding $5 \mu \mathrm{l}$ of Annexin $\mathrm{V}$ and $5 \mu \mathrm{l}$ propidium iodide $(50 \mu \mathrm{g} / \mathrm{ml})$, the cells were incubated at room temperature for 5 min in the dark using the FITC Annexin V Apoptosis detection kit (BD Biosciences). Cell apoptosis was analysed by flow cytometry (FACSCalibur FCM; Becton-Dickinson, San Jose, CA, USA).

Western blotting. Cells were washed with PBS and lysed in Lysis-M reagent supplemented with complete mini-protease inhibitor cocktail tablets (Roche Diagnostics, Indianapolis, IN, USA). The equivalent of $60 \mu \mathrm{g}$ of protein was separated on a $12 \%$ gel and then transferred onto a PVDF membrane (Millipore, Billerica, MA, USA). After blocking with 5\% $(\mathrm{w} / \mathrm{v})$ non-fat milk for $2 \mathrm{~h}$, the membranes were incubated with the following antibodies: Bax, Bcl2, GRP78, Chop, LC3, Beclin1 (1:000; Abcam, Cambridge, UK) and GAPDH (1:200; Santa Cruz Biotechnology, Inc., Dallas, TX, USA) overnight at $4^{\circ} \mathrm{C}$. Next, the membranes were incubated with a goat-anti-rabbit secondary antibody for $2 \mathrm{~h}$ at room temperature. Signals were visualised using the ChemiDoc ${ }^{\mathrm{TM}} \mathrm{XRS}+$ Imaging system (Bio-Rad Laboratories, Inc., Hercules, CA, USA).

Immunofluorescence assay. SGC-7901 cells were seeded in 6 -well plates at $3 \times 10^{5}$ cells/well and incubated for $12 \mathrm{~h}$. The cells were treated with various concentrations KFX or without KFX for $48 \mathrm{~h}$. After treatment, the cells were washed with PBS three times, fixed with $4 \%$ paraformaldehyde in PBS for 20 min, permeabilised with $0.5 \%$ Triton X-100 in PBS for $15 \mathrm{~min}$ and incubated with $5 \%$ bovine serum albumin (BSA) in PBS for $1 \mathrm{~h}$ to block nonspecific antibody binding at $37^{\circ} \mathrm{C}$. After blocking with $1 \%$ BSA for $1 \mathrm{~h}$, the cells were incubated with anti-CHOP (1:100; Santa Cruz Biotechnology, Inc.) antibody. Then, the cells were incubated with goat-anti-rabbit secondary fluorescence antibody for $2 \mathrm{~h}$ at room temperature. 
The nuclei were stained with DAPI. Fluorescence images were obtained using a positive position fluorescence microscope or confocal laser microscope (both from Nikon).

Transmission electron microscopy (TEM) image. After fixation in $2.5 \%(\mathrm{w} / \mathrm{v})$ glutaraldehyde overnight, cells were post-fixed in $2 \%(\mathrm{v} / \mathrm{v})$ osmium tetroxide and blocked with $2 \%(\mathrm{v} / \mathrm{v})$ uranyl acetate. Following dehydration in a series of acetone washes, the cells were embedded in Araldite. Semi-thin sectioning and toluidine blue staining were performed to observe the location. Finally, ultra-thin sections of at least three blocks per sample were cut and observed using a Hitachi TEM.

Statistical analyses. Statistical analyses were carried out using SPSS 20.0 statistical software (SPSS, Inc., Chicago, IL, USA). All values are presented as the means \pm standard error of the mean (SEM). Statistical evaluation of the data was performed using one-way analysis of variance (ANOVA) and Dunnett's post hoc test. $\mathrm{P}<0.05$ was considered to indicate a statistically significant difference.

\section{Results}

KFX inhibited cell growth and migration in human gastric cancer cell line SGC-7901. In the CCK-8 assay, the SGC-7901 cells were treated with KFX for 24,48 and $72 \mathrm{~h}$ at different concentrations $(0.1,0.25,0.5,1,2,5 \mu \mathrm{g} / \mathrm{ml})$ to evaluate the optimised KFX treatment on the inhibition of cell growth. In each group, $1 \mu \mathrm{g} / \mathrm{ml}$ of $\mathrm{KFX}$ showed substantial inhibition in SGC-7901 cells, whereas there was no concentration dependence over $1 \mu \mathrm{g} / \mathrm{ml}$ (Fig. 1). From the results of the cell viability test, KFX showed the strongest inhibition on SGC-7901 cell survival at $48 \mathrm{~h}$, while the cancer cell inhibition of KFX treatment at $24 \mathrm{~h}$ was much lower than that at $48 \mathrm{~h}$. However, there was no difference at 48 and $72 \mathrm{~h}$. Thus, in the CCK-8 analysis, $48 \mathrm{~h}$ of KFX treatment was the optimal administration time. Consistent with the CCK- 8 results, the scratch assay revealed that KFX could achieve the strongly inhibition on cell migration at $24 \mathrm{~h}$. However, the inhibition on cell migration at $48 \mathrm{~h}$ was similar or even stronger than that at $24 \mathrm{~h}$. Considering the optimized drug bioavailability, the treatment time at $48 \mathrm{~h}$ was better than $24 \mathrm{~h}$ (Fig. 2). The semi-quantification bar chart was show in Fig. 2. According to those two assays, the optimum treatment concentration $(1 \mu \mathrm{g} / \mathrm{ml})$ and time $(48 \mathrm{~h})$ for the KFX anticancer effect on SGC-7901 cells were used in the following studies.

KFX induced apoptosis in human gastric cancer cell line $S G C$-7901. SGC-7901 cells were treated with various concentrations of KFX and apoptosis was investigated using a FITC Annexin $\mathrm{V}$ apoptosis detection kit using flow cytometry. Compared with the control, treatment of SGC-7901 cells with different doses of KFX could induce different stages of apoptosis. Among all the experimental groups, the cells showed a progressive increase in apoptosis with increasing amounts of KFX treatment. In detail, the cells treated with $1 \mu \mathrm{g} / \mathrm{ml}$ of KFX showed nearly 2.53- and 2.75-fold higher apoptosis than those treated with $0.5 \mu \mathrm{g} / \mathrm{ml} \mathrm{KFX}$ and $0.1 \mu \mathrm{g} / \mathrm{ml} \mathrm{KFX}$, respectively, suggesting the strongest and most effective tumour inhibition at $1 \mu \mathrm{g} / \mathrm{ml}$ of KFX (Fig. 3A). Furthermore, the apoptosis effect

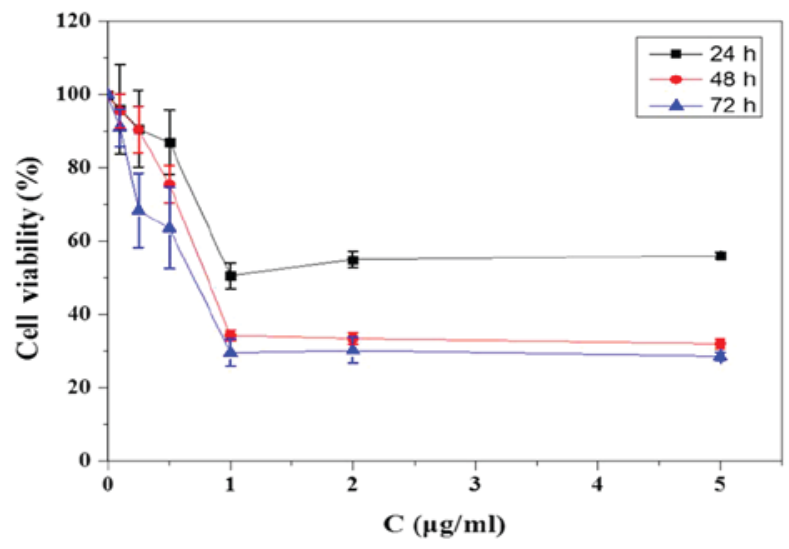

Figure 1. KFX-inhibited cell activity. CCK8 assay results of SGC-7901 cell activity after treatment with KFX for 24,48 and $72 \mathrm{~h}$ at different concentrations. KFX, Kangfuxin.

of KFX was validated by western blot analysis. Compared with the control, both 0.1 and $1 \mu \mathrm{g} / \mathrm{ml}$ of KFX upregulated the expression of Bax and downregulated the expression of Bcl-2, and the $1 \mu \mathrm{g} / \mathrm{ml} \mathrm{KFX}$ treatment group had the strongest apoptosis effect compared to the $0.1 \mu \mathrm{g} / \mathrm{ml} \mathrm{KFX}$ treatment group (Fig. 3B). The quantitative analysis of the western blot results are shown in Fig. 3C and D.

KFX promoted SGC-7901 cells apoptosis through activating ER stress. It is known that the ER can induce ER stress marker proteins (such as GRP78 and phospho-eIF2a), which upregulates the expression of CHOP (16-18). CHOP can influence the expression of $\mathrm{Bcl}-2$ family proteins and inhibit the anti-apoptotic protein expression of Bcl-2 (19). Regarding this study, many studies have proven that many TCMs inhibit cancers via activating ER stress (20-23). Therefore, we hypothesized that ER stress was involved in the anti-cancer effect of KFX and whether ER stress was associated with the mechanism of KFX-induced apoptosis in SGC-7901 cells. We examined the expression of ER stress proteins in the cells after treatment with KFX (Fig. 4). The Western blot results showed that the expressions of CHOP, GRP78 and caspase-12 were increased with $\mathrm{KFX}$ treatment group, and the $1 \mu \mathrm{g} / \mathrm{ml} \mathrm{KFX}$ group was stronger than $0.1 \mu \mathrm{g} / \mathrm{ml} \mathrm{KFX}$ group (Fig. 4A). To further confirm that KFX promoted apoptosis through ER stress, the ER stress inhibitor 4-PBA (2 mM) was used (24). 4-PBA is a chemical chaperone that contributes to protein folding and trafficking within the ER, alleviating ER stress and acting as an ER stress inhibitor (24). After exposure to 4-PBA for $6 \mathrm{~h}$, the expressions of CHOP, GRP78 and caspase-12 were significantly suppressed by 4-PBA, whereas these protein expressions were significantly restored after KFX treatment (Fig. 4C). Quantitative analysis of the western blot analysis is shown in Fig. 4B and D. In the immunofluorescence results, the CHOP expression in the cells in the 4-PBA+KFX group was stronger than the 4-PBA group (Fig. 4E). All these results demonstrated that KFX could reverse the expressions of the 4-PBA-inhibited ER-related proteins. ER-stress played a critical role in the KFX anticancer effect.

KFX activated the autophagy in SGC-7901 cells. In addition to ER stress, autophagy may also be involved in SGC-7901 

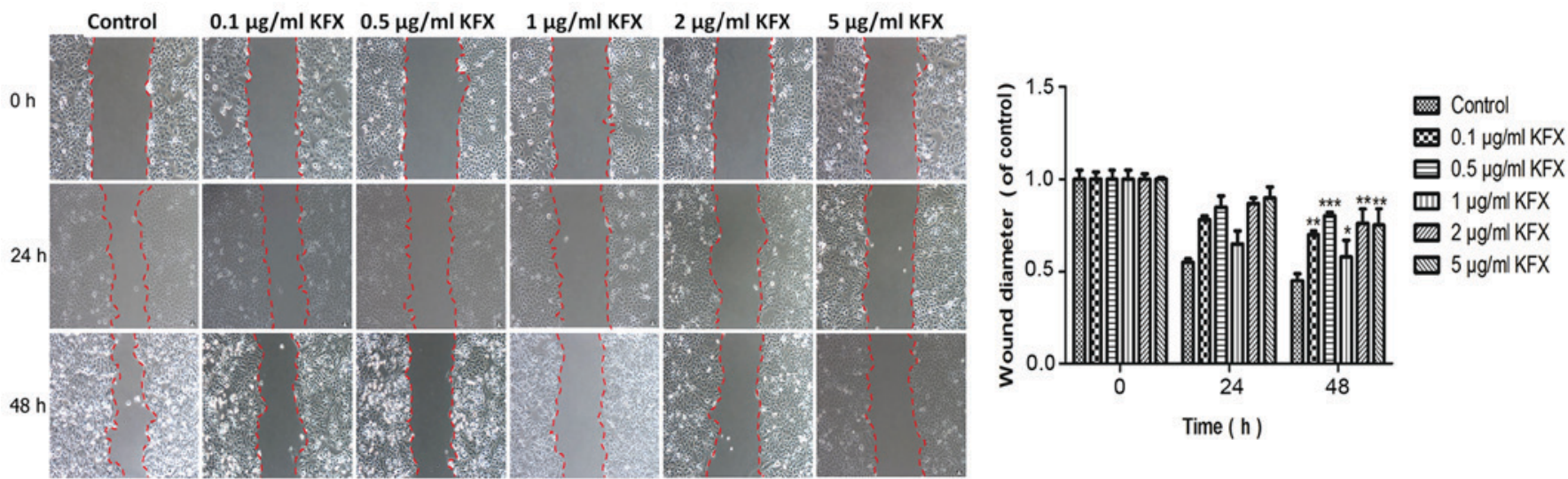

Figure 2. KFX-inhibited cell migration. Scratch assay results showed that the strongest inhibition of cell migration in the $1 \mu \mathrm{g} / \mathrm{ml} \mathrm{KFX}$ group. Data are shown as the means $\pm \mathrm{SD}, \mathrm{n}=3$. Student's t-test compared to control, ${ }^{*} \mathrm{P}<0.05,{ }^{* *} \mathrm{P}<0.01,{ }^{* * *} \mathrm{P}<0.001$ vs. control. KFX, Kangfuxin.
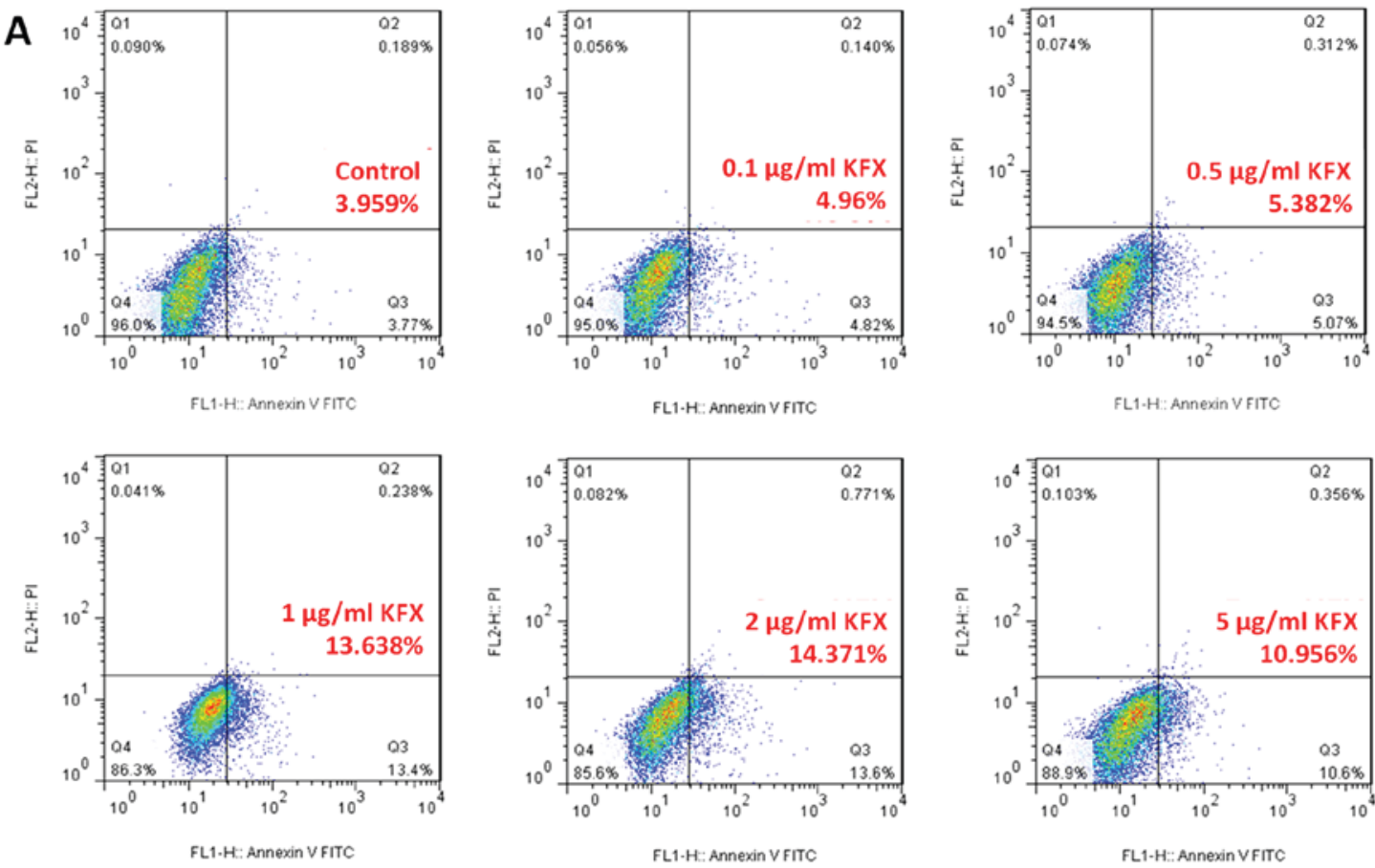

B

C

D
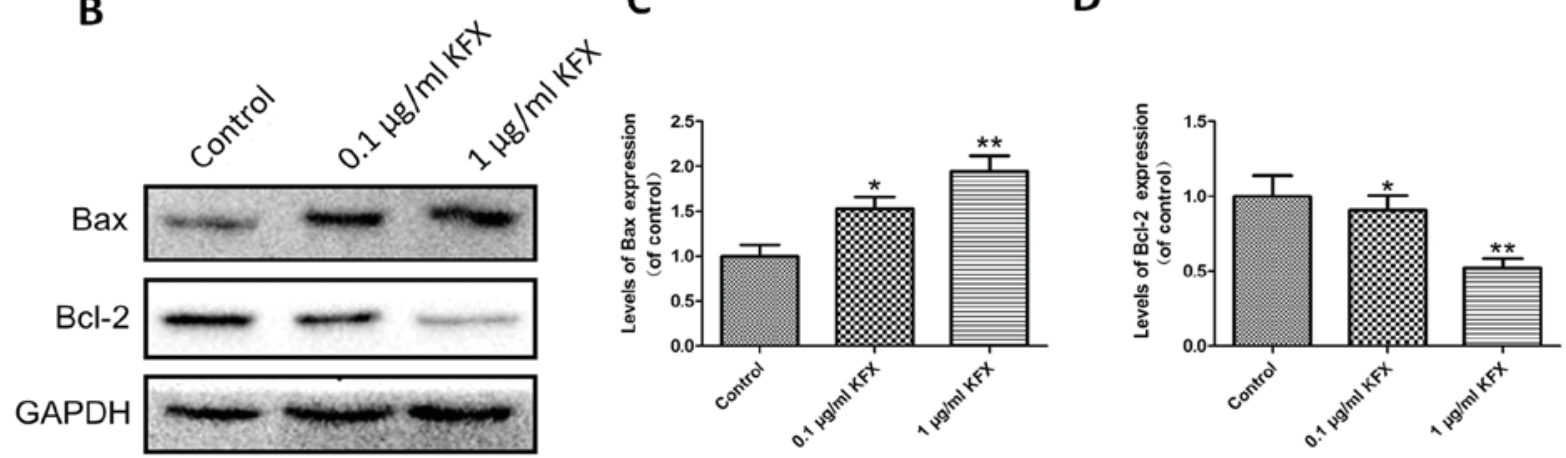

Figure 3. KFX-induced cell death of the human gastric cancer cell line SGC-7901. (A) SGC-7901 cell apoptosis was determined by flow cytometry after treatment with $\operatorname{KFX}(0,0.1,0.5,1,2,5 \mu \mathrm{g} / \mathrm{ml})$ for $48 \mathrm{~h}$. (B) Western blot results of apoptosis-related proteins Bax and Bcl-2. GAPDH was used as internal control. (C and D) Western blot results were calculated and represented as the percent of the control. All experiments were repeated three times. Data are shown as the means $\pm \mathrm{SD}, \mathrm{n}=3 .{ }^{*} \mathrm{P}<0.05,{ }^{* * *} \mathrm{P}<0.01$ vs. control. KFX, Kangfuxin.

cell apoptosis after KFX treatment. TEM images verified that KFX activated autophagy. As shown in Fig. 5A, the
KFX-treated group had many autophagosomes compared with the control group. These data revealed that KFX 

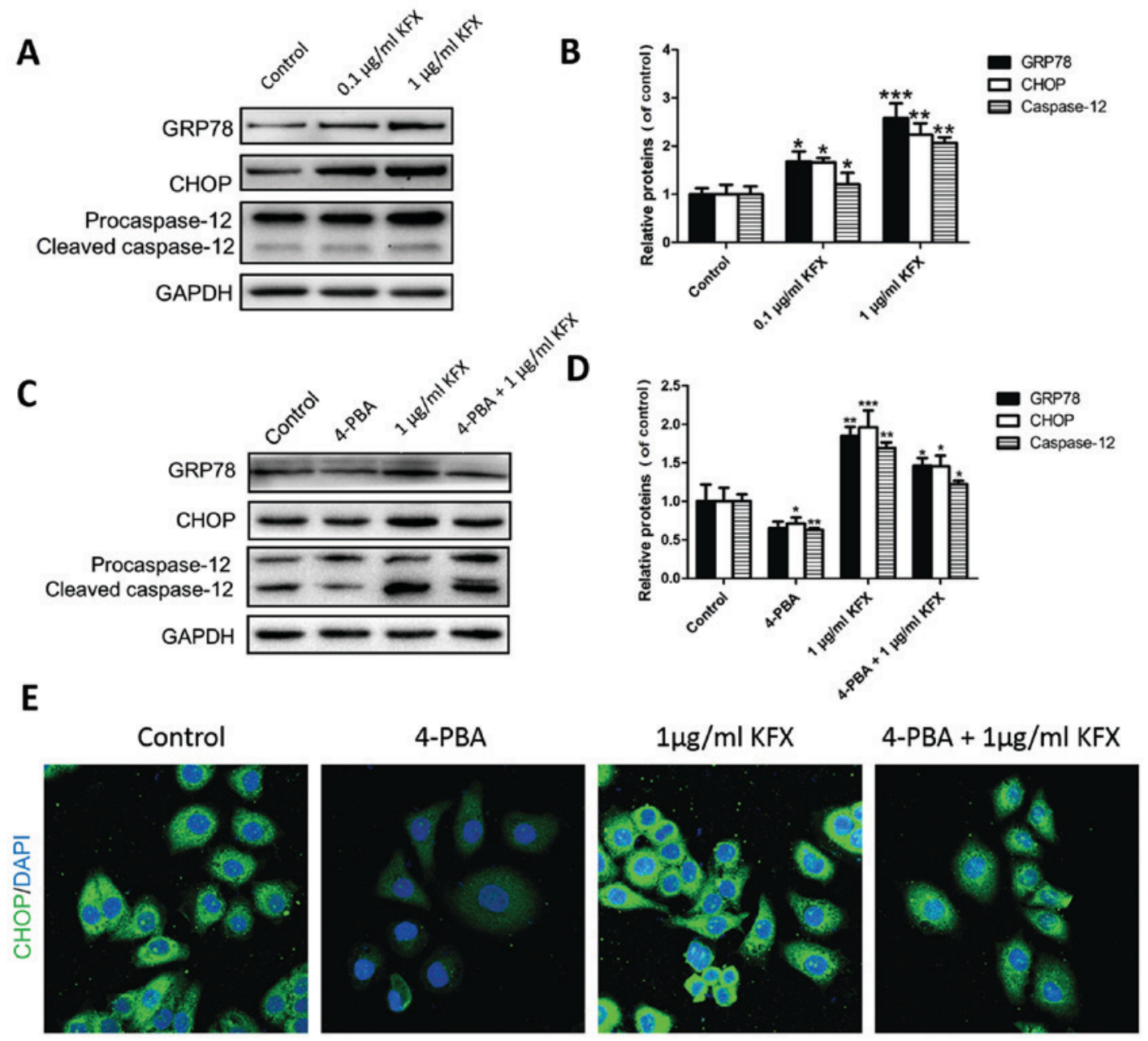

Figure 4. KFX accelerated the SGC-7901 cell apoptosis by upregulating ER stress. (A and B) Western blot analysis of the GRP78, CHOP and caspase-12 protein expressions. After the KFX treatment, the expressions of GRP78, CHOP and caspase-12 were significantly upregulated compared with the control group. GAPDH was used as the loading control and for band density normalization. (C and D) Western blot analysis of GRP78, CHOP and caspase-12 protein expression after treatment with the ER stress inhibitor 4-PBA. We found that expression of GRP78, CHOP and caspase-12 were significantly upregulated compared with the control group and, on the basis of giving $1 \mu \mathrm{g} / \mathrm{ml} \mathrm{KFX}$, the protein expression reversed. (E) Images of CHOP immunofluorescence in different groups. All experiments were repeated three times. Data are shown as the means $\pm \mathrm{SD}, \mathrm{n}=3$. Student's t-test compared to control, ${ }^{*} \mathrm{P}<0.05$, ${ }^{* *} \mathrm{P}<0.01$, ${ }^{* * *} \mathrm{P}<0.001$ vs. control. KFX, Kangfuxin; ER, endoplasmic reticulum; GRP78, glucose-regulated protein 78; CHOP, C/EBP homologous transcription factor; 4-PBA, 4-phenylbutyric acid.

could activate autophagy in SGC-7901 cells, resulting in an increase in autophagosomes in cells. In addition, we further examined the expression of the protein Beclin-1, which is a key protein in autophagy. SGC-7901 cells were treated with 0.1 and $1 \mu \mathrm{g} / \mathrm{ml} \mathrm{KFX}$ for $48 \mathrm{~h}$. As shown in Fig. 5B, the expression of Beclin-1 was significantly upregulated in the KFX groups compared with the control group. Moreover, autophagosome formation is mediated by the conjugation systems composed of ATG proteins, which culminate in the conjugation of ATG12 to ATG5 and conversion of a soluble form of microtubule-associated protein 1 light chain 3 (LC3)-I to another bound form, LC3-II (25). Thus, the ratio of LC3-II/LC3-I is a marker of autophagy in some studies $(26,27)$. The ratio of LC3-II/LC3-I in the KFX-treated groups was significantly higher than the control group, and the $1 \mu \mathrm{g} / \mathrm{ml} \mathrm{KFX}$ group showed the greatest effect (Fig. 5B and C). Furthermore, the autophagy activation in SGC-7901 cells was suppressed when treated with the ER stress inhibitor 4-PBA. Interestingly, the expressions of Beclin-1 and LC3-II/LC3-I were slightly increased in
4-PBA+KFX group (Fig. 5D). These results illustrated that KFX induces apoptosis through activation of ER stress and autophagy. However, the KFX autophagy effect may be controlled by ER stress activation. Quantitative analysis of the Western blot is shown in Fig. 5C and E.

\section{Discussion}

Gastric carcinoma causes the second highest number of cancer-related deaths in the world. Currently, surgical removal of the stomach is the only curative clinical treatment, but non-surgical cancer therapies are always the best treatment for patients. Many studies have worked on agents for inhibiting gastric cancer in rodents; however, the exact mechanisms of those agents remain unclear. As a TCM, KFX is the organic extract of Periplaneta americana and is widely used in the treatment of gastric ulcer in patients. To our surprise, the present study found that KFX could promote SGC-7901 cell apoptosis, reflecting the anti-cancer potential in gastric carcinoma. Based on this phenomenon, studies of the further 
A

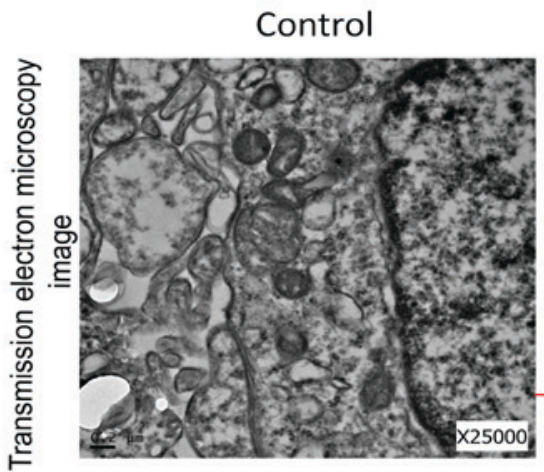

$1 \mu \mathrm{g} / \mathrm{ml} \mathrm{KFX}$

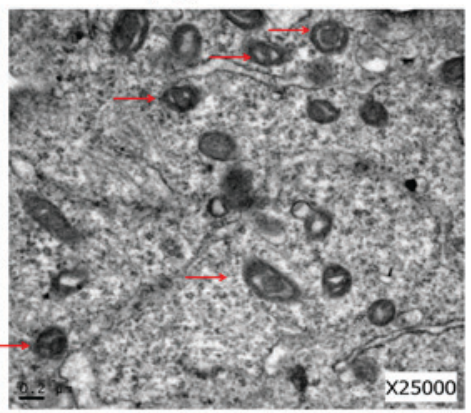

B

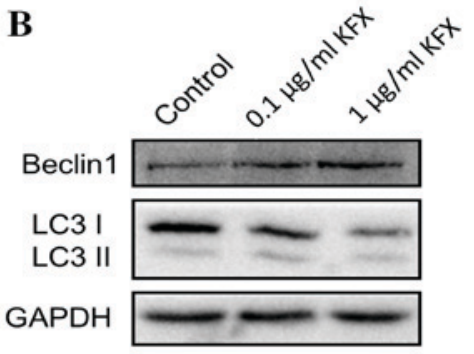

C

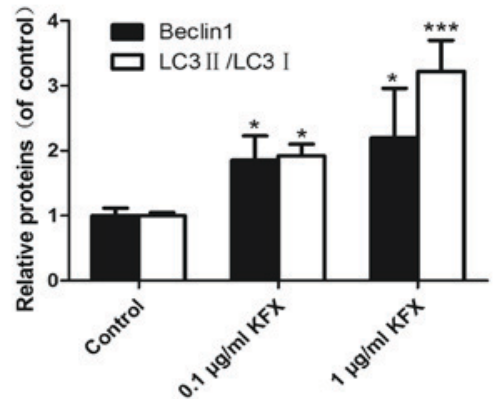

E

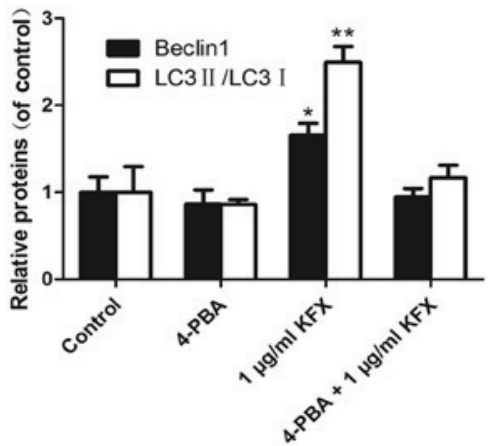

Figure 5. KFX accelerated the SGC-7901 cells apoptosis by activating autophagy. (A) Transmission electron microscopy (TEM) image results showed that autophagosomes (red arrow) were significantly increased in the KFX group compared to the control group. (B and C) Western blot analysis of Beclin-1 and LC3 protein expression. Beclin-1 and LC3 were significantly up-regulated in the KFX treated groups compared with the control group. GAPDH was used as the loading control and for band density normalisation. (D and E) Western blot analysis of Beclin-1 and LC3 protein expression after using ER stress inhibitor 4-PBA. The expression of Beclin-1 and LC3 were significantly up-regulated in the KFX-treated groups compared with the control group. All experiments were repeated three times. Data are shown as the means $\pm \mathrm{SD}, \mathrm{n}=3$. Student's t-test compared to control, ${ }^{*} \mathrm{P}<0.05,{ }^{* *} \mathrm{P}<0.01,{ }^{* * * *} \mathrm{P}<0.001$ vs. Control. Kangfuxin

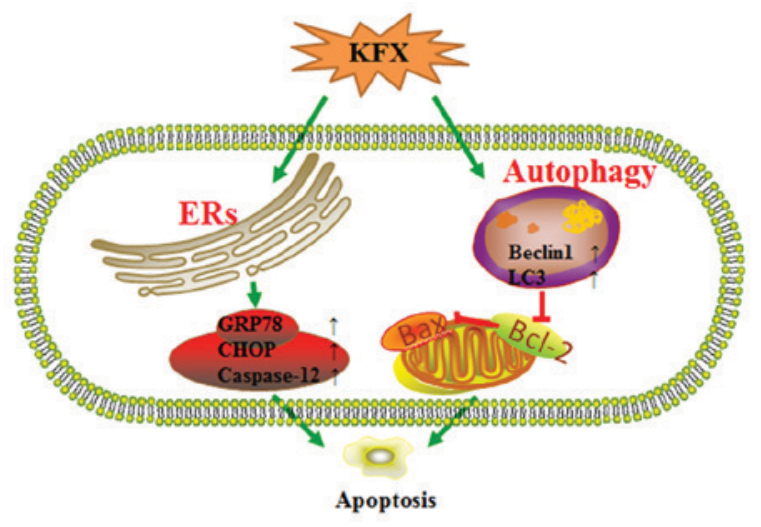

Figure 6. A diagram illustrating the KFX pro-apoptosis effect on SGC-7901 cells. Both autophagy and ER stress were activated after treatment with KFX in SGC-7901 cells, which aims to either inhibit cell migration or further accelerate SGC-7901 cell apoptosis. ERs plays a central role in promoting SGC-7901 cell apoptosis, which was induced by KFX. Autophagy-induced expression of Beclin-1 and LC3 activation contributed to SGC-7901 cell death, which was promoted by KFX. mechanisms of KFX in SGC-7901 cells were performed in this study.

Many studies have demonstrated that cancer cell apoptosis is related to ER stress-mediated autophagy $(13,28)$. It is known that ER stress induces the misfolding of the ER lumen as well as unfolded protein aggregation and calcium ion balance disorder. ER overload of caspase-12 could mediate the apoptosis signalling pathways (14). Zhang et al study showed that the targeting of apoptotic mechanisms had a close correlation with the ER stress pathway (21). Meanwhile, autophagy is a membrane process that results in the transport of cellular contents to lysosomes for degradation (29). A previous study indicated that ER stress and autophagy were involved in the apoptosis induced by cisplatin in human lung cancer cells (15). Thus, both ER stress and autophagy appear to be critical for general cell homeostasis as well as tumourigenesis and chemo-resistance. Furthermore, studies proved that the cooperation of autophagy and ER stress could achieve cancer 
inhibition in particular environments (30-33). The process of ER stress has been tightly linked to autophagy. Therefore, we hypothesized that ER stress and autophagy were involved in regulating the KFX-induced apoptosis in SGC-7901 cells.

In this study, KFX promoted the apoptosis of SGC-7901 cells, as characterized by decreased cell activity accompanied by the inhibition of cell migration. The result basically confirmed that KFX inhibited cancer cell growth in vitro and showed the potential anti-cancer effects on gastric carcinoma. In the underlying molecular mechanisms, KFX accelerated ER stress as demonstrated by the upregulated expressions of ER stress-related proteins, such as CHOP, GRP78, and caspase-12 (Fig. 6), which was consistent with the activated apoptosis effects of KFX in SGC-7901 cells. To test the critical role of ER stress from KFX, the ER stress inhibitor 4-PBA was used to suppress the ER stress-related proteins. In particular, 4-PBA inhibited ER stress-related proteins were upregulated by treatment with KFX. As a result, the KFX promoted SGC-7901 cell apoptosis and was closely related to the activation of ER stress.

Previous studies have reported that autophagy could induce apoptosis. Apoptosis and autophagy are both tightly regulated biological processes that play a central role in tissue homeostasis, development, and disease (34). In addition, many agents aggravate ER stress, leading to increased autophagic activity $(33,35)$. Autophagy is an evolutionarily conserved, dynamic and lysosome-mediated process that involves the sequestration and delivery of cytoplasmic material to the lysosome, where it is degraded and recycled (36). In theory, autophagy may contribute to promoting cell survival, either by removing the damaged organelles and toxic cell metabolites or providing the nutrients necessary for cell survival during nutrient-limiting conditions. Conversely, autophagy may also promote cell death through excessive self-digestion of cell components. Despite recent advances in the understanding of its molecular mechanisms and biological functions, it is still unclear whether autophagy acts as a cell survival or cell death pathway or both (31-34). In our study, the treatment of KFX increased the expression of autophagy-related proteins compared with the control, which showed the same tendency with the promotion of apoptosis and the activation of ER-stress by KFX treatment (Fig. 6). However, KFX could not preserve the autophagy activation in cells whose ER-stress action was suppressed by 4-PBA. Therefore, we concluded that KFX promoted apoptosis through activation of autophagy, whereas KFX-induced autophagy was followed by the promotion of ER stress.

In conclusion, KFX can be a promising and safe anticancer agent against gastric carcinoma, which can promote apoptosis through activating ER stress and autophagy (Fig. 6). ER stress may be the critical mechanism for the anti-cancer effects of KFX on gastric carcinoma and the secondary effects on autophagy. However, the specific interaction between these still need to be further explored. In the present study, we explored the potential anticancer effects of KFX and its related cancer mechanism. As a promising anti-cancer agent, further studies will be performed, including its effects on normal cell lines or other cancerous cell lines and, further, its effects on lung cancer in vivo. The further roles of KFX will be learned in our future studies.

\section{Acknowledgements}

This study was supported by crosswise tasks from Good Doctor Pharmaceutical Group (KJHX1505), research fund for the doctoral program of High Education by Ministry of Education of China (grant no. 20133321120005, Cui-Tao Lu), Zhejiang Provincial Program for the Cultivation of Highlevel Innovative Health Talents (Ying-Zheng Zhao), 151 Talent Project of Zhejiang province and 551 Talent Project of Wenzhou (Ying-Zheng Zhao), Key Support of High Level Talent Innovation and Technology Project of Wenzhou (Zhao Ying-Zheng), Zhejiang Provincial Foundation for Health Department (grant nos. 2015ZDA023 and 2016KYA136), Medicine Grant from Wenzhou Bureau of Science and Technology (grant no. Y2014730), School Talent Start Fund of Wenzhou Medical University (grant no. QTJ15020).

\section{References}

1. Li Y, Li B, Xiang CP, Zhang Y, Li YY and Wu XL: Characterization of gastric cancer models from different cell lines orthotopically constructed using improved implantation techniques. World J Gastroenterol 18: 136-143, 2012.

2. Zou P, Xia Y, Chen T, Zhang J, Wang Z, Chen W, Chen M, Kanchana K, Yang S and Liang G: Selective killing of gastric cancer cells by a small molecule targeting ROS-mediated ER stress activation. Mol Carcinog 55: 1073-1086, 2016.

3. Honda K and Jacobson JS: Use of complementary and alternative medicine among United States adults: The influences of personality, coping strategies, and social support. Prev Med 40: 46-53, 2005.

4. Wang XY, He ZC, Song LY, Spencer S, Yang LX, Peng F, Liu GM, Hu MH, Li HB, Wu XM, et al: Chemotherapeutic effects of bioassay-guided extracts of the American cockroach, Periplaneta americana. Integr Cancer Ther 10: NP12-NP23, 2011.

5. Efferth T, Li PC, Konkimalla VS and Kaina B: From traditional Chinese medicine to rational cancer therapy. Trends Mol Med 13: 353-361, 2007.

6. Alves RR, Lima HN, Tavares MC, Souto WM, Barboza RR and Vasconcellos A: Animal-based remedies as complementary medicines in Santa Cruz do Capibaribe, Brazil. BMC Complement Altern Med 8: 44, 2008.

7. Bonnemain B: Helix and Drugs: Snails for Western Health Care From Antiquity to the Present. Evid Based Complement Alternat Med 2: 25-28, 2005.

8. Castro ML, Vilela WR, Zauli RC, Ikegaki M, Rehder VL, Foglio MA, de Alencar SM and Rosalen PL: Bioassay guided purification of the antimicrobial fraction of a Brazilian propolis from Bahia state. BMC Complement Altern Med 9: 25, 2009.

9. Yang Z, Liu Y, Liao J, Gong C, Sun C, Zhou X, Wei X, Zhang T, Gao Q, Ma D and Chen G: Quercetin induces endoplasmic reticulum stress to enhance cDDP cytotoxicity in ovarian cancer: Involvement of STAT3 signaling. FEBS J 282: 1111-1125, 2015.

10. Szegezdi E, Logue SE, Gorman AM and Samali A: Mediators of endoplasmic reticulum stress-induced apoptosis. EMBO Rep 7: 880-885, 2006.

11. Kaufman RJ: Orchestrating the unfolded protein response in health and disease. J Clin Invest 110: 1389-1398, 2002.

12. Zhu SP, Wang ZG, Zhao YZ, Wu J, Shi HX, Ye LB, Wu FZ, Cheng Y, Zhang HY, He S, et al: Gelatin nanostructured lipid carriers incorporating nerve growth factor inhibit endoplasmic reticulum stress-induced apoptosis and improve recovery in spinal cord injury. Mol Neurobiol 53: 4375-4386, 2016.

13. Ogata M, Hino S, Saito A, Morikawa K, Kondo S, Kanemoto S, Murakami T, Taniguchi M, Tanii I, Yoshinaga K, et al: Autophagy is activated for cell survival after endoplasmic reticulum stress. Mol Cell Biol 26: 9220-9231, 2006.

14. Zhang XY, Zhang TT, Song DD, Zhou J, Han R, Qin ZH and Sheng R: Endoplasmic reticulum chaperone GRP78 is involved in autophagy activation induced by ischemic preconditioning in neural cells. Mol Brain 8: 20, 2015. 
15. Shi S, Tan P, Yan B, Gao R1, Zhao J, Wang J, Guo J, Li N and Ma Z: ER stress and autophagy are involved in the apoptosis induced by cisplatin in human lung cancer cells. Oncol Rep 35: 2606-2614, 2016.

16. Yoshida T, Shiraishi T, Nakata S, Horinaka M, Wakada M Mizutani Y, Miki T and Sakai T: Proteasome inhibitor MG132 induces death receptor 5 through CCAAT/enhancer-binding protein homologous protein. Cancer Res 65: 5662-5667, 2005.

17. Jiang CC, Chen LH, Gillespie S, Kiejda KA, Mhaidat N, Wang YF, Thorne R, Zhang XD and Hersey P: Tunicamycin sensitizes human melanoma cells to tumor necrosis factor-related apoptosis-inducing ligand-induced apoptosis by up-regulation of TRAIL-R2 via the unfolded protein response. Cancer Res 67: 5880-5888, 2007.

18. He Q, Lee DI, Rong R, Yu M, Luo X, Klein M, El-Deiry WS, Huang Y, Hussain A and Sheikh MS: Endoplasmic reticulum calcium pool depletion-induced apoptosis is coupled with activation of the death receptor 5 pathway. Oncogene 21: 2623-2633, 2002.

19. Bouman L, Schlierf A, Lutz AK, Shan J, Deinlein A, Kast J, Galehdar Z, Palmisano V, Patenge N, Berg D, et al: Parkin is transcriptionally regulated by ATF4: Evidence for an interconnection between mitochondrial stress and ER stress. Cell Death Differ 18: 769-782, 2011.

20. Zhu J, Chen M, Chen N, Ma A, Zhu C, Zhao R, Jiang M, Zhou J, Ye L, Fu $\mathrm{H}$ and Zhang X: Glycyrrhetinic acid induces G1-phase cell cycle arrest in human non-small cell lung cancer cells through endoplasmic reticulum stress pathway. Int J Oncol 46 : 981-988, 2015.

21. Zhang YS, Shen Q and Li J: Traditional Chinese medicine targeting apoptotic mechanisms for esophageal cancer therapy. Acta Pharmacol Sin 37: 295-302, 2016.

22. Kim B, Kim HS, Jung EJ, Lee JY, K Tsang B, Lim JM and Song YS: Curcumin induces ER stress-mediated apoptosis through selective generation of reactive oxygen species in cervical cancer cells. Mol Carcinog 55: 918-928, 2016.

23. Kim HS, Lim JM, Kim JY, Kim Y, Park S and Sohn J: Panaxydol, a component of Panax ginseng, induces apoptosis in cancer cells through EGFR activation and ER stress and inhibits tumor growth in mouse models. Int J Cancer 138: 1432-1441, 2016.

24. Zhang W, Chen L, Shen Y and Xu J: Rifampicin-induced injury in L02 cells is alleviated by 4-PBA via inhibition of the PERK-ATF4-CHOP pathway. Toxicol In Vitro 36: 186-196, 2016.

25. Tork OM, Khaleel EF and Abdelmaqsoud OM: Altered cell to cell communication, autophagy and mitochondrial dysfunction in a model of hepatocellular carcinoma: Potential protective effects of curcumin and stem cell therapy. Asian Pac J Cancer Prev 16: 8271-8279, 2015.
26. Ma K, Huang MY, Guo YX and Hu GQ: Matrine-induced autophagy counteracts cell apoptosis via the ERK signaling pathway in osteosarcoma cells. Oncol Lett 12: 1854-1860, 2016.

27. Wu L, Maimaitirexiati X, Jiang Y and Liu L: Parkin regulates mitochondrial autophagy after myocardial infarction in rats. Med Sci Monit 22: 1553-1559, 2016.

28. Liang C, Li H, Zhou H, Zhang S, Liu Z, Zhou Q and Sun F: Recombinant Lz-8 from Ganoderma lucidum induces endoplasmic reticulum stress-mediated autophagic cell death in SGC-7901 human gastric cancer cells. Oncol Rep 27: 1079-1089, 2012.

29. Xiong HY, Guo XL, Bu XX, Zhang SS, Ma NN, Song JR, Hu F, Tao SF, Sun K, Li R, et al: Autophagic cell death induced by 5-FU in Bax or PUMA deficient human colon cancer cell. Cancer Lett 288: 68-74, 2010.

30. Li X, Zhu F, Jiang J, Sun C, Zhong Q, Shen M, Wang X, Tian R, Shi C, Xu M, et al: Simultaneous inhibition of the ubiquitin-proteasome system and autophagy enhances apoptosis induced by ER stress aggravators in human pancreatic cancer cells. Autophagy 12: 1521-1537, 2016.

31. Xie WY, Zhou XD, Yang J, Chen LX and Ran DH: Inhibition of autophagy enhances heat-induced apoptosis in human non-small cell lung cancer cells through ER stress pathways. Arch Biochem Biophys 607: 55-66, 2016.

32. Xie WY, Zhou XD, Li Q, Chen LX and Ran DH: Acid-induced autophagy protects human lung cancer cells from apoptosis by activating ER stress. Exp Cell Res 339: 270-279, 2015.

33. Sharma K, Ishaq M, Sharma G, Khan MA, Dutta RK and Majumdar S: Pentoxifylline triggers autophagy via ER stress response that interferes with Pentoxifylline induced apoptosis in human melanoma cells. Biochem Pharmacol 103: 17-28, 2016.

34. Pattingre S, Tassa A, Qu X, Garuti R, Liang XH, Mizushima N, Packer M, Schneider MD and Levine B: Bcl-2 antiapoptotic proteins inhibit Beclin 1-dependent autophagy. Cell 122: 927-939, 2005

35. Periyasamy P, Guo ML and Buch S: Cocaine induces astrocytosis through ER stress-mediated activation of autophagy. Autophagy 12: 1310-1329, 2016.

36. Wang K, Liu R, Li J, Mao J, Lei Y, Wu J, Zeng J, Zhang T, $\mathrm{Wu} \mathrm{H}$, Chen L, et al: Quercetin induces protective autophagy in gastric cancer cells: Involvement of Akt-mTOR- and hypoxia-induced factor $1 \alpha$-mediated signaling. Autophagy 7 : 966-978, 2011. 